\title{
Di norma, oggi non si procede in ordini sparsi
}

\section{Kevin De Vecchis}

PUBBLICATO: 11 GIUGNO 2019

\section{Quesito:}

Alcuni lettori ci segnalano l'uso della locuzione in ordine sparso alla forma plurale nel ritornello della canzone I tuoi particolari, presentata al Festival di Sanremo 2019 dal cantautore romano Niccolò Moriconi, in arte Ultimo. Il passo in questione è il seguente: "se solamente Dio inventasse / delle nuove parole potrei dirti che / siamo soltanto bagagli / viaggiamo in ordini sparsi". Le domande pervenuteci riguardano la correttezza di tale forma.

\section{Di norma, oggi non si procede in ordini sparsi}

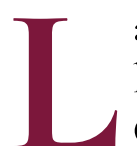

a locuzione avverbiale in ordine sparso è un sintagma preposizionale formato da più unità tra loro separate [preposizione + sostantivo + aggettivo] che costituiscono, pero, una polirematica. Come tale, è invariabile e, dunque, non potrebbe essere declinata alla forma plurale. La locuzione si è diffusa all'inizio in àmbito militare per indicare il modo di avanzare dei soldati a distanza l'uno dall'altro (cfr. Zingarelli 20I9). Il GDLI sotto la voce ordine riporta: "milit. Modo di disporre milizie, truppe, mezzi militare terrestri, navali, aerei secondo i dettami dell'arte o delle tecniche della guerra, i criteri della tattica o le particolari decisioni di un comando in funzione di una determinata operazione bellica (ordine sparso, serrato, denso, lungo, obliquo, diretto, inverso, semplice, composto, naturale, ecc.)". Come esempio si cita un passo di Ardengo Soffici (I879-1964), in cui la preposizione è articolata:

\footnotetext{
Risonavano le trombe di fantaccini, comandati dai loro ufficialinell'ordine sparso (A. Soffici, Autoritratto di un artista italiano nel quadro del suo tempo, in Id., Opere, vol. VII, Firenze, Vallecchi, I968, tomo 2, p. 315).
}

La locuzione ha assunto in seguito anche un valore più generico. Essa significa infatti 'separatamente' (Zingarelli 20I9), 'qua e là, ciascuno per suo conto' (GDLI). A tal proposito il GDLI segnala un passo di Giorgio Bassani:

Avevamo voltato per una viottola di terra battuta, finendo poi a passeggiare in ordine sparso lungo il desolato arenile che si stendeva ai piedi della rocca (G. Bassani, Il giardino dei Finzi-Contini, Torino, Einaudi, 1962, p. II).

In rete la forma in ordine sparso è di fatto esclusiva [in Google 655.00o risultati al o2/05/2019]. Inoltre, si possono trovare esempi piu lontani nel tempo nella sezione Libri del motore di ricerca Google;sono infatti presenti attestazioni già a partire dalla metà del XIX secolo, come la seguente:

$\S$ 9I. Formazione normale di una truppa in ordine sparso. Modificazioni risultanti dalla natura del terreno. L'ordine di battaglia di una truppa in ordine sparso è quale lo prescrive il Regolamento d'esercizio per la scuola de' cacciatori. Or dunque la disposizione di una truppa in ordine sparso può assimilarsi ad una disposizione d'avamposti suscettiva al par di questa di modificarsi all'atto pratico in ragione della natura del suolo (Istruzione sulle operazioni secondarie della guerra ad uso degl'Uffiziali dell'Esercito, redatta per cura del Corpo reale di Stato Maggiore ed approvata dal Ministro della Guerra, Torino, Tip. scolastica di Sebastiano Franco e figli, r855, p. 99). 
La forma plurale in ordini sparsi, invece, non è attestata in nessun dizionario. Gli esempi presenti in Google (22.400 risultati al $02 / 05 / 2019$ ) rimandano esclusivamente alla canzone citata in apertura. In Google Libri abbiamo tuttavia cinque attestazioni: tre della fine dell'Ottocento, una dell'inizio del Novecento e una più recente del 2015. Si riportano qui di seguito, dalla più antica alla più recente:

Fin dalle età più remote, i combattimenti tutti presentano l'azione precipua, che in essi esercitano le grandi masse di fanteria, e l'azione, per così dire secondaria della fanteria stessa adoprata in ordini sparsi, a frazioni concatenate l'une all'altre e designate a proteggere e preparare la prima, coll'esplorare i movimenti nemici, e col francheggiare le ali della massa principale, proteggendola da improvvisi assalti e col preparare gli attacchi della massa medesima coi fuochi individuali, frequenti, repentini, ben nutriti e diretti ad offendere in particolare maniera i capi avversari ("Rivista militare italiana. Raccolta mensile di scienza, arte e storia militari dell'esercito italiano", s. III, XX, I875, 2, p. 404).

A questo aveva già da pezza rivolto il suo studio Epaminonda; aveva seguito con occhio vigile tutti i progressi fatti dall'arte militare, s'era persuaso di ciò, che in difficili condizioni si potrebbe pure ottenere, raggruppando in ordini sparsi, le schiere (Ernst Curtius, Storia greca, trad. di Joseph Müller e Gaetano Oliva, vol. 3, Torino-Roma, Loescher, I884, p. 306).

I fucili a ripetizione e le moderne artiglierie ànno tolto assai all'impeto delle baionette, cambiando gli ordini serrati in ordini sparsi, per i quali i terreni frastagliati e coperti diventano preziosi ausiliari (Giovanni Battista Plini, L'Italia nella politica europea, Napoli, Jovene, I89o, p. 33).

Inoltre è avvenuta anche una sempre più accentuata tendenza, nella tattica della fanteria, di muovere a sbalzi, in ordini sparsi, cercando sempre di usufruire nel miglior modo delle accidentalità del campo di battaglia ("Rivista di artiglieria e genio", II, I906, p. 344).

E facile scontrarsi. È difficile trovarsi. Siamo troppi e tutti in ordini sparsi. Non è vero che non voglio farmi prendere. La verità è che poi dopo non mi so difendere. Ho un carattere che consuma le persone. Corrode i miei amici (E., L'altro me, s.l., Narcissus.me, 2015, e-book).

Dunque, quattro delle attestazioni risalgono a un periodo in cui la locuzione non si era ancora cristallizzata, mentre l'ultima è in un testo pubblicato solo in rete, la cui appartenenza allo standard andrebbe attentamente verificata. Come valutare, allora, l'uso di in ordini sparsi da parte di Ultimo? Innanzitutto bisogna considerare che il sintagma compare allinterno di un testo scritto per essere cantato. Spesso nelle canzoni, anche per motivi di musicalità e di rima, i cantautori si prendono delle libertà di scrittura non propriamente in linea con la norma linguistica, ma più vicine all'uso parlato, e non sempre è facile capire se si tratti di scelte intenzionali o meno. Si potrebbero citare numerosi esempi, come l'uso del che polivalente cantato da Jovanotti in Ragazzo fortunato (I992): "Sono un ragazzo fortunato / perché m’hanno regalato un sogno / sono fortunato / perché non c'è niente che ho bisogno"; oppure il mancato accordo tra soggetto plurale e verbo singolare in Un senso di Vasco Rossi (2004): "Voglio trovare un senso a tante cose / anche se tante cose un senso non ce l'ha". È vero che in questo ultimo caso si potrebbe interpretare tante cose come complemento oggetto e un senso come soggetto (la violazione della norma starebbe allora nell'elisione l'ha, impossibile con il clitico plurale le), ma semanticamente è poco plausibile anche in rapporto ai versi precedenti dove al posto di tante cose compaiono i seguenti sostantivi sera, vita, storia, voglia, situazione e condizione).

In questo caso, il plurale in ordini sparsi potrebbe essersi prodotto per via dell'inferenza di un soggetto plurale sottointeso 'noi' e del predicativo 'bagagli'. A livello di senso, invece, il plurale potrebbe rimandare a un uso enfatico che il cantante vuole esprimere per sottolineare che ognuno di noi ha il suo "ordine sparso", il proprio modo, casuale e disordinato, di viaggiare nella sua dimensione, diversamente da tutti gli altri. Tale interpretazione potrebbe trovare conferma nel video musicale 
della canzone, in cui il protagonista viaggia in luoghi ed epoche diverse alla ricerca della sua amata, precedendo, appunto, "in ordine sparso", lontano anche dallo spazio in cui si trova il cantante, che siede al pianoforte in una sala ampia. Inoltre, il testo è caratterizzato da un lessico quotidiano ("La tua voce al mattino che grida bu") e da un uso colloquiale della lingua, molto piu vicino a quella parlata che scritta: si veda come esempio l'uso di te come pronome soggetto invece ditu, tratto tipico di alcuni italiani regionali, tra i quali quello di Roma, da dove il cantante proviene: "E da tempo che cammino e / sento sempre rumori dietro me / poi mi giro pensando che ci sei te / e mi accorgo che oltre a me non so che c'e'".

In conclusione, l'uso corretto della locuzione resta in ordine sparso, così come confermano i dizionari consultati e gli esempi trovati in rete. Tuttavia, dato il contesto in cui si inserisce il nostro in ordini sparsi, qui nella inusuale collocazione con il verbo viaggiare, si può concedere la giusta dose di tolleranza alla libertà espressiva che il cantante si è concesso.

\section{Cita come:}

Kevin De Vecchis, Di norma, oggi non si procede in ordini sparsi , "Italiano digitale", 2019, IX, 2019/2 (aprile-giugno)

DOI: $10.35948 / 2532-9006 / 2020.3123$

Copyright 2019 Accademia della Crusca

Pubblicato con licenza creative commons CC BY-NC-ND 